\title{
21 Präpositionalobjektsätze
}

1 Präpositionalobjektsätze im System der Komplemente und Supplemente: funktionale und kategoriale Einordnung

2 Formen von Präpositionalobjektsätzen

3 Korrelatkonstruktionen

4 Topologische Eigenschaften von Konstruktionen mit Präpositionalobjektsätzen

5 Zusammenfassung

6 Literatur

\section{Präpositionalobjektsätze im System der Komplemente und Supplemente: funktionale und kategoriale Einordnung}

Präpositionalobjekte (POe) werden in valenzgrammatischen Beschreibungen, unabhängig vom jeweils zugrundeliegenden valenztheoretischen Konzept, wie das Subjekt und die Objekte in obliquen Kasus einhellig zu den durch die Valenz eines Regens gebundenen Konstituenten des Satzes gezählt (vgl. Ágel 2000, Breindl 1989, 2006, Eroms 1981, 2000). Sie haben somit den Status von „Ergänzungen“ resp. „Komplementen“, sind allerdings keine „Aktanten“ im Sinne von Tesnière (1959), der hierunter nur Kasus-NPen rechnet, während präpositional oder konjunktional angebundene Konstituenten bei ihm zu den Zirkumstanten zählen. In der Tat zeigen valenzgebundene PPen manche Eigenschaften von adverbialen PPen, insbesondere eine im Vergleich zu Kasusmorphemen deutlich ausgeprägtere Eigensemantik der Präposition. Das macht sie zu notorischen Problemkandidaten für die Einordnung in die valenztheoretische „Zweiklassengesellschaft“ (Ágel 2000) von Ergänzungen und Angaben. Mit einem multidimensionalen, graduellen Valenzkonzept wie dem von Jacobs (1994, als Manuskript zirkulierend seit 1987) kann man ihrem etwas hybriden Status besser gerecht werden. Auf dieser Basis wurden sie in Breindl (1989) und Zifonun/Hoffmann/Strecker et al. (1997, im Folgenden GDS) beschrieben; unter dem Aspekt ihrer Grammatikalisierung aus adverbialen PPen bei Hundt (2001) und Roustila (2005). Der Sonderstatus der Präpositionalobjekte findet auch bei ihren satzförmigen Realisierungen ein Echo, da das Auftreten pronominaladverbialer Korrelate wie darin, davon, darum etc. einige Besonderheiten gegenüber den anderen Komplementsätzen mit sich bringt (vgl. die Beispiele in (1)). Diese Korrelate haben semantisch 
eine den Korrelaten von adverbialen Subjunktoren (wenn, weil, statt dass etc.) vergleichbare Wirkung, insofern sie die inhaltliche Relation des Nebensatzes zum Matrixsatz transparent machen, da ihr präpositionaler Bestandteil mehr autonome Bedeutung mitbringt (vgl. Breindl 1989: 39f.) als ein Korrelat-es, das nicht nur voll grammatikalisiert, sondern auch ambig in Bezug auf die syntaktische Funktion ist, vgl. (1). Die Beispiele unter (2a-f) illustrieren die unterschiedlichen kategorialen Realisierungsmöglichkeiten für Präpositionalobjektsätze: propositionsdenotierende dass-Sätze (2a), $o b$-Sätze (2b) und w-Sätze (2c) (die letzteren beiden werden traditionell als indirekte Fragesätze zusammengefasst), uneingeleitete Verbzweitsätze (2d), Infinitivphrasen (2e) und gegenstandsdenotierende $w$-Verbletztsätze (Freie Relativsätze), die zu jedem Valenzträger auftreten können (2f).

(1) a. Normalerweise wird (es) nicht bekannt gegeben, wer den Antrag bearbeitet.

(Subjektsatz)

b. Normalerweise geben wir (es) nicht bekannt, wer den Antrag bearbeitet. (Akkusativobjektsatz)

(2) a. Ein Antragsteller beschwert sich (darüber), dass er schon zwei Monate auf einen Bescheid wartet.

b. Die Bearbeitung hängt davon ab, ob die nötigen Unterlagen vorliegen.

c. Der Antragsteller erkundigt sich (danach), wer den Antrag bearbeitet.

d. Er beharrt darauf, es lägen nicht alle Unterlagen vor.

e. Der Sachbearbeiter will sich (darum) bemühen, die Angelegenheit zügig zu erledigen.

f. Glaub doch, woran du glauben willst.

Für die Forschung zu Präpositionalobjektsätzen sind neben Grammatiken vor allem die valenzgrammatisch orientierten Monographien von Eroms (1981) und Breindl (1989) einschlägig, ferner Arbeiten zu Komplementsätzen ganz allgemein oder zu speziellen Formtypen (Beneš 1979, Zint-Dyhr 1981, Reis 1997, Pittner 1995, 2003, Berman 2003, Oppenrieder 2006); vergleichende Hinweise finden sich auch in den beiden Monographien von Bausewein (1990) zu Akkusativobjektsätzen und Oppenrieder (1991) zu Subjektsätzen. Schließlich sind auch Arbeiten zu Korrelatkonstruktionen heranzuziehen (Fabricius-Hansen 1981, Zitterbart 2002, Boszák 2009).

Präpositionalobjektsätze haben im System der Komplementsätze sowohl Gemeinsamkeiten mit Akkusativobjektsätzen (s. Abschnitt 1.1) als auch mit Adverbialsätzen (s. Abschnitt 1.2). Formal können sie prinzipiell durch die gleichen Formtypen realisiert werden wie Subjekt- und Objektsätze (s. Abschnitt 2). Dabei 
können oder müssen in Abhängigkeit von lexikalischen und strukturellen Bedingungen Korrelate auftreten (s. Abschnitt 3). Auch in ihren Stellungseigenschaften heben sich Präpositionalobjektsätze im System der Komplementsätze ab (s. Abschnitt 4).

\subsection{Präpositionalobjektsätze im System der Komplementsätze}

Die Möglichkeit einer satzförmigen Realisierung eines Präpositionalobjekts ist nicht im gleichen Maße eine spezifische lexikalische Eigenschaft des Valenzträgers, wie dies für die Subkategorisierung für eine bestimmte Komplementfunktion überhaupt (Subjekt, Akkusativ-, Dativ-, Genitiv-, Präpositionalobjekt) gilt. Freie Relativsätze etwa wie (2f) können uneingeschränkt jede Komplementstelle realisieren, da sie Individuen bzw. Kollektive von Individuen denotieren können. Alle anderen satzförmigen Realisierungen von Präpositionalobjekten, also dassSätze wie (2a), ob-Sätze wie (2b), „propositionsfundierte“ $w$-Sätze (GDS 1997: 2266) wie (2c), Verbzweitsätze wie (2d) und Infinitivphrasen wie (2e), sind auf Valenzträger beschränkt, die an der entsprechenden Argumentstelle eine Proposition zulassen. Diese Eigenschaft kann in der Regel für semantisch definierte Subklassen von Valenzträgern geltend gemacht werden und ist unabhängig von der Komplementfunktion, die der Valenzträger an der betreffenden Argumentstelle fordert. Satzförmige Realisierungen erlauben z.B. die direkten Objekte (,Zweitaktanten") von Kommunikations- und Kognitionsverben (berichten (AkkO), berichten von (PO), glauben an (PO)), von intentionalen Verben (beabsichtigen (AkkO), abzielen auf (PO)), die Komplemente von faktiven Verben (bedauern (AkkO), bedauerlich/ärgerlich/erfreulich sein (Subj.), sich ärgern über/sich freuen über (PO)) oder von implikativen Verben (verhindern (AkkO), hindern an (PO)). Für solche Subklassen können tendenziell auch zulässige oder nicht zulässige Realisierungsformen der Komplementsätze vorausgesagt werden (s. Abschnitt 2).

Präpositionalobjekte realisieren meist semantische Rollen, die auch ein propositionales Argument zulassen. Weitgehend ausgeschlossen sind propositionale Argumente für Benefizienten- und Komitativ-Rollen, die typischerweise von belebten Sachverhaltsbeteiligten ausgefüllt werden, wie in s. (ab)melden/anrufen bei, schicken an, sich verabreden/treffen/unterhalten mit. Präpositionalobjekte mit diesen semantischen Rollen alternieren zum Teil mit Dativobjekten (jdm. schicken/an jdn. schicken). Keine satzförmige Realisierung erlauben auch PPen mit konkret zu interpretierenden lokalen und direktionalen Rollen (auspacken aus, kommen über, kommen an, kommen aus). Es handelt sich hier durchweg um strittige Fälle der Valenztheorie, für die aufgrund der mit valenzfreien adverbialen PPen identischen Kodierung ohnehin eine Klassifikation als Supplemente an- 
gemessener scheint (vgl. aber Schumacher et al. (2004), wo sie durchweg als „K $\mathrm{K}_{\text {prp }}$ " klassifiziert werden).

Unter den Komplementsätzen sind Präpositionalobjektsätze am ehesten mit Akkusativobjektsätzen vergleichbar, schon weil sie wie diese Zweit- oder Drittaktanten sind und gleiche semantische Rollen realisieren können. Dativobjekte haben in aller Regel Individuendenotate, sodass sie (außer als Freie Relativsätze) kaum satzförmig auftreten, und Genitivobjekte treten zwar als Sätze auf, sind im Gegenwartsdeutschen aber sehr selten. Subjekte wiederum realisieren eher andere semantische Rollen wie Agens oder Experiencer, die wie Dativobjekte Individuendenotate haben. Im Vergleich zu Akkusativobjekten und Subjekten erlauben Präpositionalobjekte wesentlich häufiger satzförmige Realisierungen. In E-VALBU, der Internet-Version von Schumacher et al. (2004), beträgt der Anteil satzförmig realisierbarer Komplemente bei Präpositionalobjekten 1/3, bei Akkusativobjekten 1/7 und bei Subjekten nur 1/16. Das erklärt sich zum Teil dadurch, dass Präpositionalobjekte Grammatikalisierungen von Adverbialia sind, von denen sie sich gerade durch die Nicht-Konkretheit unterscheiden (sich stützen auf, abzielen auf, hervorgehen aus). Akkusativobjekte wiederum weisen im Sinne des graduellen Transitivitätskonzepts von Hopper/Thompson (1980) eine höhere Transitivitätsbindung an den Prädikatsausdruck auf als Präpositionalobjekte, und das prototypische Direkte Objekt eines transitiven Verbs denotiert eher ein von der Prädikatshandlung affiziertes Individuum und eben keine Proposition.

Die Identifikation der - kasusunmarkierten - Komplementsätze als Subjekt-, Akkusativobjekt-, Präpositionalobjektsätze etc. erfolgt auf der Basis ihrer paradigmatischen Austauschbarkeit mit einem kasusmarkierten Pronomen bzw. einer ProPP als „Leitform“ (s. GDS 1997: 1071 im Anschluss an Engel 1977). Gegen diese funktionale Parallelisierung von Kasusobjekten und Nebensätzen setzt Berman (2003) eine Klassifikation der Nebensätze, die sich an deren Position orientiert. Dadurch werden topikalisierte und extraponierte Nebensätze u.U. unterschiedlich analysiert und letzteren eine spezielle sententiale Komplementfunktion COMP zugewiesen, für die eine weitere funktionale Differenzierung unnötig ist. Für eine solche Annahme scheinen - abgesehen von syntaxtheoretischen Erwägungen und der leichteren Anschließbarkeit an die Diskussion außerhalb der germanistischen Linguistik - für das Deutsche zunächst die Existenz einer Handvoll Verben zu sprechen, bei denen ein satzförmiges Komplement nach dem Leitformprinzip nicht oder nicht eindeutig auf ein Kasus-Komplement zurückführbar ist. Das gilt für einige Verben, die nur Infinitivphrasen zulassen (sich weigern, geruhen, gedenken, beabsichtigen, suchen, sich anstrengen, nicht umhin können). Sie werden in Engel (1994), in der GDS (1997) und in Schumacher et al. (2004) als separate Klasse „Verbativkomplemente“ ausgewiesen. 
(2) a. Sie weigert sich, zum Arzt zu gehen/??dagegen/*dessen/*das.

b. Streng dich an, unter die ersten $10 \mathrm{zu}$ kommen/?? dazu.

Diese Verben lassen sich mit ihrer semantischen Komponente der Intentionalität zur Modalverbperipherie (scheinen, pflegen, versprechen) stellen; sie bilden einen Übergang zwischen der Bildung von Verbalkomplexen und der Einbettung von Infinitivphrasen mit Komplementstatus (vgl. GDS 1997: 1073; zu sich weigern Breindl 1989: 153).

Auch die Verbletzt- und Verbzweitsätze zu bestimmten Kommunikationsverben lassen sich schwer funktional zuordnen. Diese Kommunikationsverben können einerseits einstellig gebraucht werden, wenn die kommunikative Absicht ist, bestimmte Aspekte der Form der Äußerung hervorzuheben. Sie können aber auch um einen satzförmigen Ausdruck erweitert werden, der den Äußerungsinhalt, das Kommunikat, kodiert (3a). Eine Substitution durch eine akkusativische Leitform ist hier mitunter nicht möglich (3b), bisweilen aber die durch ein Pronominaladverb (3c).

(3) a. Er \{schimpfte/zeterte/nörgelte/maulte/hauchte/prahlte/renommierte/ warnte\} dass er sich das nicht gefallen lasse/er lasse sich das nicht gefallen.

b. ${ }^{\star} \operatorname{Er}\{$ schimpfte/zeterte/nörgelte/maulte/hauchte/prahlte/renommierte/ warnte\} das/diese Äußerung.

c. Er \{schimpfte/zeterte/?nörgelte/?maulte\} darüber/\{prahlte/renommierte\} damit/warnte davor.

In (3c) ergibt sich jedoch eine Bedeutungsverschiebung vom assertierten Aussageeinhalt zum Kommentar über einen präsupponierten Sachverhalt. Korrelatlose dass-Sätze und Verbzweitsätze zu diesen Verben sind somit keine Realisierungen eines Präpositionalobjekts. Die „Leitformprobe“ funktioniert bei ihnen nicht, weil es gerade ihre Funktion ist, Äußerungen wiederzugeben. Auch sie werden deshalb in Engel (1994) den Verbativergänzungen zugeschlagen (bzw. $E_{\text {prop }}$ bei Eroms 2000; vgl. auch Wolf 2003: 409). Dagegen lässt sich ins Feld führen, dass bei den semantisch einfacheren Kommunikationsverben eine akkusativische Leitform immer möglich ist (das habe ich gesagt/geäußert/mitgeteilt/gesprochen) und man bei den semantisch komplexeren Kommunikationsverben eine Valenzerweiterung zum analogen Muster annehmen kann. Für die V2-Sätze in dieser Umgebung könnte man mit Reis (1997) auch von relativer Selbständigkeit und parataktischem Anschluss ausgehen, für die Verbletztsätze mit dass und $o b$ ist das allerdings nicht möglich.

Verbletzt- und Verbzweitsätze zu Valenzträgern mit alternierenden Valenzrealisierungen bereiten generell Identifikationsprobleme. Gerade Präpositional- 
objekte sind davon häufig betroffen, da sie im Umbau des Kasussystems seit dem Frühneuhochdeutschen eine zentrale Rolle spielen: Sie kompensieren den Schwund von Objektsgenitiven (sich einer Sache/an etw. erinnern, sich einer Sache/für etw. schämen, sich einer Sache/von etw. vergewissern, einer Sache/an etw. gewohnt sein) und sind beim Umbau von ergativischer (unpersönlicher) zu akkusativischer Kodierung beteiligt, die schon im Ahd. einsetzt (vgl. Dal 1966: 166f): mich wundert es (Gen.) > es (Nom.) wundert mich > ich wundere mich darüber. Meist ist im Gegenwartsdeutschen aber das Genitivobjekt die markierte Variante, sodass man einen korrelatlosen Komplementsatz wie Ich freue mich, sie zu sehen wohl eher als Realisierung eines Präpositionalobjekts als eines Genitivobjekts analysieren wird. Damit liefert gerade das Verhalten der Präpositionalobjekte Argumente gegen eine nicht nach der syntaktischen Funktion differenzierte einheitliche „Nebensatzfunktion“ COMP wie in Berman (2003): Mit dem prinzipiell immer möglichen Auftreten des Korrelats kann die syntaktische Funktion bei PO-Sätzen transparent und eindeutig markiert werden, - anders als beim Auftreten des funktionsambigen Korrelats es/das. Darüber hinaus bringt das Auftreten der Präposition im Korrelat gegenüber dem korrelatlos angeschlossenen Nebensatz oft eine zusätzliche inhaltliche Komponente ins Spiel, die exakt der bei PPförmiger Kodierung entspricht, sodass die Alternation der Komplementstelle mit einem Lesartenwechsel beim Valenzträger einhergeht.

\subsection{Abgrenzung gegenüber Adverbialsätzen}

$\mathrm{Zu}$ den PPen, deren Einordnung regelmäßig in valenztheoretischen Arbeiten problematisiert wird, zählen solche mit kausalen (leiden an), komitativen (sich verabreden/unterhalten/verloben/einigen/auseinandersetzen mit) und instrumentalen Rollen (sich brüsten/prahlen/angeben/kontern mit) sowie PPen mit abstrakten Goal- bzw. Source-Rollen (führen zu, resultieren in/aus, hinauslaufen/ übertragen/abzielen auf, hervorgehen aus). Diese PPen unterscheiden sich rollensemantisch und in der Form nicht von ihren valenzfreien adverbialen Pendants. Aber auch bei satzförmiger Kodierung dieser Rollen können die Formen zusammenfallen und Präpositionalobjektsätze mit einem Korrelat können die Oberflächenform von Adverbialsätzen haben. In den folgenden Paaren sind die Nebensätze in den (a)-Beispielen jeweils aufgrund des Vorliegens der Valenzrelation „Notwendigkeit“ eher Komplement-Kandidaten, während die (weglassbaren) Nebensätze in den (b)-Beispielen traditionell eher als Supplement klassifiziert würden. 
(4) a. Der Hundefänger köderte den Hund damit, dass er ihm ein Leckerli anbot.

b. Damit, dass einer einem Hund Leckerli anbietet, wird er nicht automatisch zum Tierfreund.

(5) a. Die Kosten für die Dokumentenbearbeitung und -prüfung begründen sich dadurch, dass ein zweites oder drittes Paar Augen die Arbeit überprüft.

b. Dadurch, dass im Herbst 2009 auf eine Preisanpassung verzichtet wurde, können die Technischen Betriebe Flawil die erhöhte Belastung für den Endkunden allerdings abfedern. (St. Galler Tagblatt 07. 01. 2010, S. 41)

Solche Beispiele über einen Kamm zu scheren, sei es als Komplemente, sei es als Supplemente, würde das Kriterium der Notwendigkeit entkräften, das in der Regel als hinreichend (wenn auch nicht notwendig) für einen Status als valenzgebunden angesehen wird (sofern man bestimmte Kontexte als nicht-diagnostisch für Weglassbarkeit berücksichtigt, vgl. Pasch 1977, Breindl 1989, Storrer 1992, GDS 1997: 1031f.). Eine Lösung besteht darin, diese instrumentalen, komitativen und kausalen PPen als eine weitere Klasse adverbialer Ergänzungen auszuweisen neben den auf Steinitz (1969) zurückgehenden lokalen, direktionalen und den durch Engel (1994) etablierten „dilativen“ Adverbialkomplementen (i.e. Maßadverbiale wie zwei Kilo wiegen, drei Stunden dauern, um 2 Grad erhöhen). Diesen Weg geht das Valenzwörterbuch von Schumacher et al. (2004), das gleich drei weitere Klassen von Adverbialkomplementen postuliert: „Mittel/Instrument“ (mit dem Finger auf etw. zeigen), „Zweck“ (zur Einreise einen Pass brauchen), „Zusammenhang“ (bei der Urteilsfindung berücksichtigen). Eine Alternative besteht darin, wie Storrer (1992) auf einer kommunikativen Ebene unterschiedliche Grade von kommunikativer Notwendigkeit anzunehmen. Festzuhalten bleibt, dass Konstituenten mit den genannten adverbialtypischen semantischen Rollen auf einem Kontinuum von Valenzbindung anzusiedeln sind, und zwar unabhängig davon, ob sie als PPen oder sentential realisiert sind. Ein solcher Übergang manifestiert sich auch in den Verbindungen eines pronominaladverbialen Korrelats mit einem dass-Satz (dadurch dass, dafür dass, dazu dass), die wie Vexierbilder sowohl als Korrelatkonstruktionen von PO-Sätzen als auch als (grammatikalisierte und lexikalisierte) komplexe adverbiale Subjunktoren analysierbar sind. 


\section{Formen von PO-Sätzen}

Präpositionalobjektsätze treten, wie in Abschnitt 1 gezeigt, in den gleichen Formtypen auf wie Subjekt- und Akkusativobjektsätze und die seltenen Genitivobjektsätze (vgl. Bsp. (2)). Durch welchen Formtyp ein Präpositionalobjekt realisiert werden kann, wird primär durch den Valenzträger bestimmt, kann aber durch weitere Kontextmerkmale im Matrixsatz (Negation, Satzmodus, Modalverben, fokussierende Operatoren etc.) beeinflusst werden. Diese Selektion ist deshalb nur bedingt gleichzusetzen mit der Valenzrelation der Formspezifizität (dem klassischen Rektionsbegriff), der ausschließlich eine lexikalische Eigenschaft des Valenzträgers ist.

\subsection{Subkategorisierte Verbletztsätze: dass-, ob-, w-Sätze}

Die Formtypen für Komplementsätze sind durch semantische Merkmale charakterisiert, die sich darauf beziehen, ob die Proposition im Wissensstand aller Kommunikationsteilnehmer „abgeschlossen“ ist (dass-Sätze, Verbzweitsätze, Infinitivphrasen) oder „offene“ Stellen enthält, sei es in der Polarität ( $o b$-Sätze), sei es in einzelnen Parametern ( $w$-Sätze) (vgl. Breindl 1989: 202ff., Oppenrieder 2006: 902ff., GDS 1997: 2253ff.). Prädikatsausdrücke lassen sich nach den möglichen Formtypen für eine Argumentstelle zu Klassen ordnen. Das ergibt zunächst einmal zwei „reine“ Klassen, die jeweils nur für Komplementsätze mit dem Merkmal geschlossen oder offen spezifiziert sind. $\mathrm{Zu}$ ersteren zählen die faktiven Verben bedauern, leid tun; mit einer PO-Valenz: sich freuen/sich ärgern/sich beklagen/ sich wundern über, leiden an, mit denen ein Sprecher die Wahrheit der Komplementsatzproposition präsupponiert, die somit in allen Aspekten dem Wissen der Kommunikationspartner zugänglich sein muss (6a). Auch implikative und negativ-implikative Verben wie kommen $z u$, hindern an gehören hierher, die die Wahrheit oder Falschheit der Komplementsatzproposition assertieren (6b), sowie Kognitionsverben (glauben an, rechnen mit, überzeugt sein von) (6c). Einfügung einer Negation ändert bei diesen drei Gruppen lediglich bei den implikativen Verben das Vorzeichen für die Geltung der Komplementsatzproposition, überschreibt aber in keinem Fall die spezifische Selektionsbeziehung zwischen Valenzträger und Einleiteelement.

(6) a. Ich freue mich (nicht) darüber, dass Ihnen der Entwurf gefällt/*ob Ihnen der Entwurf gefällt/*wem der Entwurf gefällt.

b. Am Ende kam es (nicht) dazu, dass die Arbeit wiederholt werden musste/ ${ }^{\star}$ ob die Arbeit wiederholt werden musste/ ${ }^{\star}$ warum die Arbeit wiederholt werden musste. 
c. Ich rechne (nicht) damit, dass Ihnen der Entwurf gefällt/*ob Ihnen der Entwurf gefällt/*wem der Entwurf gefällt.

Nur für offene Sachverhaltsbeschreibungen spezifiziert sind dagegen Verben des Fragens und Forschens wie fragen nach, sich erkundigen nach, forschen nach, gespannt sein auf, neugierig sein auf.

(7) Man erkundigte sich (nicht) danach, ob/wann/*dass der Entwurf fertig wäre.

Neben diesen reinen Prädikatklassen gibt es ,gemischte' Prädikatsausdrücke, die je nach der Ausgestaltung des Matrixsatzes Sätze beiderlei Typs zulassen. Eine entscheidende Rolle kommt dabei modalisierenden Ausdrücken zu, die aus einer geschlossenen Sachverhaltspräsentation eine offene machen können wie Negation, Fragesatzmodus, Modalverben. Ferner ist hier an Kontexte zu denken, in denen die Geschlossenheit der Proposition für den Sprecher oder für den Referenten des Matrixsatzsubjekts gilt, für andere Kommunikationsteilnehmer aber nicht alle Parameter aufgedeckt werden.

(8) a. Die Experten konnten sich nicht (darüber) einigen, ob/*dass sie es mit echten oder gefälschten Pollocks zu tun hatten.

b. Die Experten konnten sich (darüber) einigen, dass sie es mit gefälschten Pollocks zu tun hatten.

c. Konnten sich die Experten (darüber) einigen, ob sie es mit gefälschten Pollocks zu tun hatten?

d. Die Experten konnten sich (darüber) einigen, ob sie es mit gefälschten Pollocks zu tun hatten.

(9) a. Anna erinnert sich (nicht) daran, dass Maria heute Geburtstag hat/wann Maria Geburtstag hat.

b. Anna erinnert sich nicht daran, ob Maria heute Geburtstag hat/wann Maria Geburtstag hat.

c. ?Anna erinnert sich daran, ob Maria heute Geburtstag hat.

d. Nur Anna erinnert sich daran, ob Maria heute Geburtstag hat. (aber andere haben dieses Wissen nicht.)

In (8d) und (9d) gelten unterschiedliche Informationsstände für den Adressaten der Äußerung und für den Subjektsreferenten. Von diesem behauptet der Sprecher, dass er keine Unklarheit in Bezug auf die im Komplementsatz denotierte Proposition hat, deckt dies aber für den Adressaten nicht auf. Der Informationsstand des Sprechers ist dabei irrelevant.

Einen besonderen Fall stellen $w$-Sätze dar, die nicht das Merkmal Offenheit haben, da sie Indirektheitstypen zu Exklamativsätzen sind (vgl. Oppenrieder 1991: 190f.). 
(10) a. Die an der Evaluation beteiligt sind, wundern sich, wie sehr formale Aspekte im Vordergrund stehen. (Braunschweiger Zeitung, 21.11. 2006)

b. Viele Leute brüsten sich damit, wie viel sie durch hartnäckiges Feilschen um die neue Couchgarnitur oder den supermodernen Videorekorder gespart haben. (Mannheimer Morgen, 22. 07. 2000)

c. Sie ließen sich von den dargebotenen Schmankerln zum Schlemmen verleiten. So mancher wunderte sich, was man mit Polenta alles anstellen kann. (Kleine Zeitung, 11. 10. 1998)

Als Einleiteelement tritt vor allem wie, seltener auch was auf. Der Komplementsatz bringt zum Ausdruck, dass ein Aspekt seiner Bedeutung quantitativ oder qualitativ eine hohe Ausprägung hat; oft enthält der Komplementsatz graduierbare Adjektive oder Adverbien. In diesen $w$-Sätzen kann die für selbständige Exklamativsätze typische Modalpartikel doch auftreten, während in $w$-Sätzen mit offener Proposition nur die fragesatztypischen denn und wohl auftreten können.

(11) a. Dementsprechend genossen alle das kalte Bad und wunderten sich, wie gut man doch eigentlich riechen konnte. (St. Galler Tagblatt, 14. 08. 2008, S. 37)

b. Alle fragten immer wieder nur nach der Zensur im Iran, aber niemand fragte danach, was denn eigentlich zensiert wurde. (Vorarlberger Nachrichten, 13. 02. 1999, S. F14)

\subsection{Freie Relativsätze}

Präpositionalobjektsätze in der Funktion von Freien $w$-Relativsätzen zeigen die üblichen Unterschiede gegenüber den vom Valenzträger subkategorisierten w-Sätzen (Geschlossenheit vs. Offenheit der Proposition, kein Mehrfachauftreten von $w$-Ausdrücken bei Relativsätzen, Übereinstimmung von Relativum und Relativsatzfunktion). Die Kongruenzforderung nach Übereinstimmung der morphologischen Markierung des Relativums mit der syntaktischen Funktion des Relativsatzes im Matrixsatz ist für Präpositionalobjektsätze nicht verletzbar, weil sie am untersten Ende der Kasushierarchie NOM > AKK > DAT > PRÄPOSITIONALKASUS stehen, die den Spielraum für Kasusdivergenz beschreibt (s. Pittner 1995, 2003). Ein Relativum in PO-Funktion kann zwar in einem Relativsatz mit Subjekt- oder Akkusativobjektsatz auftreten wie in (12a), nicht aber ein Relativum in reinem Kasus in einem Relativsatz mit PO-Funktion wie in (12b). Um einen solchen Anschluss zu ermöglichen, muss ein pronominaler Kopf zusammen mit der Präposition auftreten. 
(12) a. Sie genießt, worüber er sich ärgert.

b. *Er ärgert sich, was sie genießt.

c. Er ärgert sich über das, was sie genießt.

Da auch die Präpositionsforderungen von Matrixsatz und Präpositionalobjektsatz übereinstimmen müssen, sind solche Konstruktionen auf identische oder allenfalls semantisch eng verwandte Prädikatsausdrücke beschränkt und deshalb entsprechend selten.

(13) a. Jeder hat das Recht zu glauben, woran er mag. (Braunschweiger Zeitung, 09. 02. 2010)

b. Dann war ich frei, zu suchen, wonach mein Herz begehrte. (St. Galler Tagblatt, 26. 10. 2009, S. 43)

\subsection{Verbzweitsätze}

Die Hauptdomäne unselbständiger Verbzweitsätze ist die Redewiedergabe, wobei es offenbar einen gewissen Spielraum gibt, was als verbum dicendi interpretierbar ist. Wenn der Verbzweitsatz selbst durch Konjunktiv und Deixisverschiebung oder durch Kennzeichnung als direktes Zitat als Kommunikat eindeutig erkennbar ist, können auch Prädikatsausdrücke auftreten, die selbst keine verba dicendi sind, sondern redebegleitende Aspekte thematisieren. Eine stilistische „Marotte“ mancher Presseorgane scheint dabei die Voranstellung solcher Verbzweitsätze vor einen Satz ohne freie Argumentstelle wie in (14b, c). Der Verbzweitsatz zeigt mit einem fallenden Akzent Selbständigkeitsmerkmale, besetzt aber das Vorfeld des nachfolgenden Verbzweitsatzes, der als intonatorischer Hintergrund erscheint.

(14) a. Er sei ein „Reformer mit Resultaten“, brüstet sich der republikanische Kandidat. (St. Galler Tagblatt, 13. 05. 2000)

b. „Alle haben jetzt die Möglichkeit, sich dort zu präsentieren“, freut sich Quartiermanager Gabriel Höfle über das breite und moderne Angebot. (Mannheimer Morgen, 08. 01. 2010, S. 29)

c. „So weit ich weiß, ist die Jugend sehr zufrieden“, wundert sich Heinz Schuh über die Kritik. (Niederösterreichische Nachrichten, 10. 03. 2010)

Fälle wie (14) passen zum Muster von Redewiedergaben, bei denen eine Umkehrung der Hierarchie von Matrixsatz und Komplementsatz stattfindet und der Matrixsatz - formal und intonatorisch reduziert - in einer beliebigen Parenthesennische des Kommunikats erscheinen kann wie in der Schiller'schen Bürgschaft „Ich bin“, spricht jener, „zu sterben bereit ...“. Ihr höherer Grad an Selbständigkeit 
zeigt sich auch darin, dass sie im Unterschied zu Präpositionalobjektsätzen mit einem Einleiteelement in der Position vor dem Matrixsatz nicht auf eine Linksversetzungskonstruktion angewiesen sind (vgl. Abschnitt 4). Sie lassen sich auch schlecht als Gliedteilsätze integrieren und sind deshalb mit dem Gliedteilsatzmuster (z.B. im Skopus eines Operators im Matrixsatz wie Fokuspartikel oder Kontrastnegation), deutlich weniger akzeptabel als dass-Sätze.

(15) a. Die Anklage stützt sich vor allem DArauf, sie habe einen Augenzeugen.

b. Die Anklage stützt sich vor allem DArauf, dass sie einen Augenzeugen habe.

PO-Verben, die mit Verbzweitsätzen auftreten sind z.B. hinweisen/verweisen/sich berufen auf, informieren/sprechen/klagen/jammern über, kontern/prahlen/spotten/drohen mit, ferner die dreiwertigen auffordern/raten/ermahnen zu, warnen vor, bitten um. Extraponierte Verbzweitsätze können oder müssen (valenzträgerabhängig) mit Korrelat auftreten.

(16) a. Wolff wies darauf/^ hin, es sei wichtig, dass auch den Kleinkindern täglich ein frisch zubereitetes warmes Essen angeboten werden könne. (Rhein-Zeitung, 13. 02. 2010)

b. Stoiber drohte erneut (damit), er werde im Bundesrat gegen den Euro stimmen, sollte Deutschland das Defizitkriterium von 3,0 Prozent nicht einhalten. (Tiroler Tageszeitung, 22. 09. 1997)

Verbzweitsätze sind alternative Realisierungen von Komplementsätzen mit einem Merkmal Geschlossenheit, also von dass-Sätzen und Infinitivphrasen. Für einige Prädikatsausdrücke, z.B. hinweisen auf (vgl. 16a und 17a,b) und bestehen auf, sind alle drei Realisierungen möglich.

(17) a. Die Ermittler weisen darauf hin, weitere, belastende Indizien zu haben. (Vorarlberger Nachrichten, 01. 12. 2000, S. A3)

b. Die DB Netz AG weist darauf hin, dass Baumaschinen im Einsatz sind. (Rhein-Zeitung, 20. 01. 2010; Kompakt)

\subsection{Infinitivphrasen}

Realisierung eines Komplementsatzes als Infinitivphrase setzt voraus, dass das getilgte Komplementsatzsubjekt von einer Konstituente des Matrixsatzes kontrolliert wird, also mit diesem referenzidentisch ist, aus dem Kontext zu ergänzen oder im Sinne des generischen man zu interpretieren ist. Letzteres ist bei unpersönlichen Verben immer der Fall (sich handeln um, ankommen auf, hinauslaufen 
auf). Bei den dreiwertigen Verben der Beeinflussung (bitten um, überzeugen von, überreden zu, warnen vor) übt im Normalfall die Objekts-NP Kontrolle aus, in Passivsätzen oder mit Modalverben ist auch Subjektkontrolle möglich. Die dreiwertigen implikativen Verben (zwingen $z u$, veranlassen zu, hindern an) lassen überhaupt nur Kontrolle durch das Objekt zu.

(18) a. Kanzlerin Angela Merkel warnte davor, Arbeitslose gegen Menschen mit Arbeit auszuspielen. (Nürnberger Nachrichten, 20. 02. 2010, S. 1)

b. Sie bat den Ausschuss darum, den Plan schon einmal in Ruhe anzuschauen. (Braunschweiger Zeitung, 11. 02. 2010)

d. Dennoch bat ich darum, doch vorrücken zu dürfen. (Braunschweiger Zeitung, 27. 01. 2010)

e. Nach Ende der Frist 1981 bat er darum, aus dem Priesterstand entlassen zu werden. (Nürnberger Nachrichten, 12. 04. 2010, S. 4)

Wie Akkusativ- und Dativobjekte (jdn. bitten um, jdm. raten zu) können auch Präpositionalalobjekte mit einer Adressatenrolle Kontrolle ausüben:

(19) a. In seinem Schlussgebet richtete der Pfarrer die Bitte an den Herrgott, uns seine Güte weiterhin zu erhalten. (St. Galler Tagblatt, 03. 08. 2000)

b. Der General appellierte an die Arbeitgeber, das freiwillige Engagement zu unterstützen. (Rheinzeitung, 29. 03. 2010)

Bei diesen dreiwertigen Verben ist eine alternative Realisierung als dass-Satz meist deutlich schlechter, wenn auch nicht ausgeschlossen. Die Präpositionalobjektsätze bei diesen Verben bezeichnen nonfaktische Sachverhalte, was sich mit der semantischen Charakteristik von dass-Sätzen, die in erster Linie assertieren oder präsupponieren, dass ein Sachverhalt der Fall ist, offenbar schlecht verträgt (vgl. Beneš 1979). Bei sich freuen wird die Lesart sich freuen auf mit nonfaktischem Komplement tendenziell eher mit einem Infinitiv konstruiert, die Lesart sich freuen über mit faktischem Komplement eher mit dass-Satz. Dennoch können Infinitive durchaus auch Komplemente zu faktiven Verben realisieren, wenn die Bedingungen für Kontrolle gegeben sind.

(20) Ich freue mich darüber, heute einen besonderen Gast begrüßen zu dürfen. Infinitivphrasen werden auch erlaubt von volitiven Verben wie bereit sein $z u$, fähig sein zu, neigen zu, sich bemühen um, sich einlassen auf, verzichten auf, streben nach, ablassen von, zögern mit, die als Verben der „Selbstbeeinflussung“ quasi die zweiwertigen Varianten der Verben des Beeinflussens darstellen. 


\section{Korrelatkonstruktionen}

Verglichen mit Subjekt- und Akkusativobjektsätzen spielen Korrelatkonstruktionen für Präpositionalobjektsätze eine wesentlich wichtigere Rolle. Korrelate sind bei Valenzträgern mit einer PO-Valenz sehr viel häufiger lexikalisch oder konstruktionsbedingt obligatorisch als bei den anderen beiden Komplementsatztypen; sie sind auch nie zur Gänze ausgeschlossen. (Zu Korrelaten allgemein s. Fabricius-Hansen (1981), Sonnenberg (1992), GDS (1997: 1474ff.), Zitterbart (2002), Boszák (2009), zu Korrelaten bei Präpositionalobjektsätzen Holmlander (1979), Breindl (1989).)

Korrelate zu Präpositionalobjektsätzen sind Pronominaladverbien der Form $d a(r)$ + Präposition und treten in zwei Varianten auf: in einer im gegebenen Satzkontext unakzentuierten Form, die bei den dar-Formen auch auf die Schwundstufe $d r$-reduziert sein kann, und in einer Form mit einem deutlich erkennbaren Akzent auf dem pronominalen Teil. Im ersten Fall kann der Satzakzent im Matrixsatz selbst liegen und der Präpositionalobjektsatz liefert Hintergrundmaterial, im zweiten Fall ist der Präpositionalobjektsatz auf jeden Fall fokal und enthält den (fallenden) Satzakzent; das Pronominaladverb hat dann einen steigenden Akzent. In Breindl (1989) werden diese Formen nach ihrer syntaktischen und prosodischen Struktur terminologisch in Analogie zum Unterschied zwischen es und das als Korrelaten bei Subjekt- und Akkusativobjektsätzen (vgl. Oppenrieder 1991) terminologisch als Platzhalter mit Gliedsatz-Akzentmuster (21a) und Bezugselement mit Gliedteilsatz-Akzentmuster (21b) voneinander unterschieden.

(21) a. Mir liegt eine ganze MENge daran, dass Sie wahrheitsgemäß antworten.

b. Wahlprognosen sind häufig ungenau. Das liegt /DAran, dass die Bürger die Sonntagsfrage nicht immer WAHRheitsgemäß beantworten.

Die beiden Formen gehen mit unterschiedlichen informationsstrukturellen, syntaktischen und topologischen Mustern einher. Beim Gliedteilsatz-Akzentmuster liegt ein engerer Fokus vor als beim Gliedsatz-Akzentmuster; nur letzeres kann voll fokale Konstruktionen realisieren.

(22) Was ist los?

a. Im Radio hat jemand davon / geSPROchen, dass eine InflaTION droht.

b. \#Im Radio hat jemand /DAvon gesprochen, dass eine InflaTION droht. 


\subsection{Kategoriale Einordnung der Korrelatkonstruktionen}

Prinzipiell lassen sich Korrelatkonstruktionen nicht ohne Weiteres zentralen syntaktischen Konstruktionsmustern zuordnen, da sie scheinbar Doppelbesetzungen einer Argumentstelle innerhalb eines komplexen Satzes sind, was gegen ein grundlegendes syntaktisches Prinzip verstößt. In der grammatischen Literatur wurden in den 80er- und 90er-Jahren etliche Lösungen diskutiert (s. den Überblick in der GDS 1997: 1487f. und Zitterbart 2002), darunter die als Doppelbelegung und die einer Attributkonstruktion. In der GDS (1979), ebenso Zitterbart (2002) und Boszák (2009), werden „Korrelatverbindungen“ - Paare aus einem phorischen Ausdruck und einem Komplementsatz - als eine Konstruktionskategorie sui generis betrachtet, die immer dann auftreten kann, wenn der Valenzträger für ein satzförmiges Komplement subkategorisiert ist. Zum syntaktischen Verhältnis zwischen den Bestandteilen einer Korrelatverbindung werden dabei aber keine Angaben gemacht. Da die phorische Funktion des Pronomens bzw. Pronominaladverbs nach der GDS aber auch in der Korrelatfunktion erhalten bleibt, läuft das letztlich doch auf eine Art Doppelbelegung innerhalb eines Satzes hinaus, die entfernte Ähnlichkeit mit Links- und Rechtsversetzung (Diesen Gauner, den kenne ich gut; ich kenne ihn gut, diesen Gauner) hat. In Breindl (1989) wurde ein referentieller Status für Korrelate mit Platzhalterfunktion abgelehnt; Platzhalter sind demnach rein formal markierte „dummy-Argumente“, die eine kohärente Abarbeitung des Mittelfelds und eindeutige Zuordnung des extraponierten Komplementsatzes erlauben, haben also eher den Status einer Spur und teilen nicht die Stellungseigenschaften referierender Proformen: Sie erlauben keine adjazente Stellung im selben Stellungsfeld und treten nur im Mittelfeld bei extraponiertem Komplementsatz auf, bei Subjektsätzen auch im Vorfeld. Dieses Auseinandertreten von formaler (valenzgesteuerter) Markierung am Platzhalter und inhaltlicher Auffüllung im Komplementsatz lässt sich gerade mit dem mehrdimensionalen Valenzkonzept von Jacobs (1994) gut erfassen. Andererseits können prinzipiell auch referierende, kataphorisch verweisende Proformen wie Platzhalter unakzentuiert auftreten, wodurch eine Kategorisierung ersterer als nicht-referierend etwas willkürlich wirkt. Die Proform hat jedenfalls in beiden Verwendungen kataphorische Verweisfunktion (vgl. 23a).

(23) a. Wer hat nicht schon davon geHÖRT, dass der vermeintliche Traumprinz sich als optischer Alptraum entpuppt hat?

b. Wer hat nicht schon davon geHÖRT: Der vermeintliche Traumprinz entpuppt sich als optischer Alptraum!

Die Alternativlösung als Attributkonstruktionen wird in Breindl (1989) nur für das „Gliedteilsatzmuster“ mit akzentuierten Korrelaten vertreten. Dafür spricht die 
Möglichkeit, dass ein stark akzentuiertes Korrelat zusammen mit dem adjazenten Komplementsatz im gleichen Stellungsfeld auftreten kann (24a). Dagegen spricht, dass die Form des Nebensatzes durch den Valenzträger und nicht wie sonst für Attribute üblich durch den Kopf der Attributkonstruktion bestimmt wird (24bc) (vgl. die Kritik in GDS 1997: 1489).

(24) a. Er hatte DArüber, ob/dass sie Vermögen hatte, nicht allzuviel nachgedacht.

b. Er hatte über die TATsache, dass/^ob sie Vermögen hatte, nicht allzuviel nachgedacht.

b. Er hatte über die FRAge, ob/^dass sie Vermögen hatte, nicht allzuviel nachgedacht.

In (24) liegt mit nachdenken über ein Valenzträger vor, der zur Charakteristik von offenen und geschlossenen Propositionen passt. Die nominalen Köpfe Tatsache und Frage sind hingegen jeweils spezifisch für einen der beiden Typen. Dieselbe Verteilung zeigt aber auch das Platzhaltermuster:

(25) a. Er hatte nicht allzuviel d(a)rüber NACHgedacht, ob/dass sie Vermögen hatte.

b. Er hatte nicht allzuviel über die Tatsache NACHgedacht, dass/*ob sie Vermögen hatte.

b. Er hatte nicht allzuviel über die Frage NACHgedacht, ob/*dass sie Vermögen hatte.

Der Unterschied ist primär informationsstruktureller Natur. Das GliedteilsatzAkzentmuster ist ein Epiphänomen der Fokussierung des Komplementsatzes, für die das Korrelat als Fokusexponent dient. Dass bestimmte Valenzträger, insbesondere sachverhaltskorrelierende, im Defaultfall ein akzentuiertes Korrelat fordern (bestehen in, liegen an, beruhen auf, führen $z u$ ) hat mit ihrem geringen eigenen Mitteilungswert und einer daraus abzuleitenden verminderten Fokussierbarkeit zu tun. In bestimmten Kontexten sind sie aber durchaus fokussierbar.

(26) Abrafax: Momentan haben wir unser Konto $(\mathrm{GmbH})$ bei einer beratergesteuerten Bank. Ein Problem dabei ist, dass sich andere Mitarbeiter grundsätzlich kaum angesprochen fühlen, wenn man Fragen oder Wünsche hat. Beatrix: Hallo, wie wäre es mit „reinen“ Internetbanken? Deren Konzept beRUHT ja gerade darauf, dass man möglichst keine Berater braucht. (http://www.wer-weiss-was.de/theme220/article4829271.html)

Das Auftreten des Gliedteilsatz-Akzentmusters bei Präpositionalobjektsätzen erweist sich somit als kontextuell bedingt; durch Fokussierungsmittel (Fokuspartikeln, Negation) kann es erzwungen werden, bei manchen Prädikatsausdrücken 
stellt es sich aufgrund ihres geringen Mitteilungswerts, der nicht allzuweit über die Existenzpräsupposition für die in Relation zueinander gebrachten Sachverhalte hinausgeht, als Defaultwert ein, kann aber durch geeignete Kontextbedingungen überschrieben werden.

\subsection{Lexikalische Bedingungen des Auftretens von Korrelaten}

Korrelate zu Präpositionalobjektsätzen sind sehr viel häufiger als bei Subj.- und AkkO-Sätzen aufgrund der Forderung des Valenzträgers obligatorisch. Das rührt daher, dass das „funktionale Plus“ (GDS 1997: 1476) von Korrelatkonstruktionen gegenüber solchen ohne Korrelat bei diesen Valenzträgern besonders vorteilhaft zur Geltung kommt.

- Die semantische Relation der Komplementsatzproposition zu der des Matrixsatzes wird durch die Setzung der Präposition, die mehr oder weniger autosemantisch, aber keineswegs völlig desemantisiert ist, transparenter als beim Auftreten des (funktionsambigen) es.

- Die Setzung der Präposition kann (als eine Art semantischer Bestandteil des Valenzträgers) die Identifikation der intendierten Lesart des Valenzträgers sichern. Gerade Prädikatsausdrücke mit einer PO-Valenz treten häufig in alternierenden Valenzmustern auf (vgl. Ágel 2000).

Auf der Basis dieser funktionalen Vorteile erklärt sich die Korrelat-Obligatorik bei den folgenden Klassen von Prädikatsausdrücken. Eine Mehrfachauflistung zeigt, dass die Faktoren auch zusammentreffen können, was auf ihre zumindest partielle Interdependenz hindeutet.

- Das Korrelat differenziert zwischen bedeutungsverschiedenen Valenzträgern mit alternierenden PO-Valenzmustern: bestehen auf/aus/in, kämpfen (sich aussprechen, plädieren, stimmen) für/gegen, sich freuen auf/über, es bringen auf/zu, liegen an/in, klagen um/über, sein bei/an/für/gegen: Mitunter ist hier nur bei einer - meist der semantisch komplexeren, merkmalhaltigeren - Lesart der Platzhalter obligatorisch, so etwa bei sich freuen auf oder sich entscheiden gegen (nicht aber bei sich freuen über, sich entscheiden für).

- Das Korrelat differenziert zwischen bedeutungsverschiedenen Valenzträgern mit alternierenden Valenzmustern und dient gleichzeitig der Identifizierung der Lesart des Valenzträgers und der Argumentstelle. Dazu zählen z.B. verba dicendi, sentiendi und putandi mit Variation zwischen Akkusativ- und PO-Valenz: denken (an), glauben (an), hoffen (auf), sprechen/erzählen (von/ über), klagen (um/über), fragen (nach), ferner semantisch unterspezifizierte, in zahlreichen Valenzmustern ausgeprägte Verben wie kommen (zu), ankom- 
men (auf), liegen (an/in), führen (zu), gehen (um), halten (für/an/von), setzen (auf), sein (für/gegen/an/bei).

- Das Korrelat kennzeichnet bei sachverhaltskorrelierenden Prädikatsausdrücken, die zwei Komplementsätze zulassen, die Funktion des PO-Komplementsatzes ( $O b$ dies der Fall ist, hängt davon ab, ob etwas anderes der Fall ist): abhängen von, etw. ändern an, sich ausdrücken in, sich auswirken auf, aufbauen auf, beruhen auf, folgen aus, führen zu, hervorgehen aus, hinauslaufen auf, hindeuten auf, handeln von, resultieren in, zusammenhängen mit. Tendenziell tritt das Korrelat hier als akzentuiertes Bezugselement auf, da der Prädikatsausdruck semantisch zu „blass“ ist, um selbst als Fokusexponent aufzutreten.

- Das Korrelat macht bei Komplementen im Übergangsbereich zu Adverbialen autosemantisch die adverbial-semantische Rolle des Präpositionalobjektsatzes kenntlich. Das betrifft erstens Komplementsätze mit instrumentaler oder komitativer Rolle zu Valenzträgern mit den Präpositionen mit und durch (beweisen/begründen/erklären mit/durch, (sich beschäftigen/abgeben/Mühe geben mit, aufschneiden/prahlen/angeben/sich wichtig tun/sich entschuldigen mit). Zweitens zählen dazu Valenzträger mit PO-Rollen, die sich auf lokale und direktionale Adverbialia zurückführen lassen (Goal-, Source-, Place-Rollen), - insbesondere mit auf und aus als direktionaler und an als lokal-statischer Präposition: (abzielen/sich beschränken/sich berufen/hinweisen/es absehen/hoffen/lauem/verfallen/sich vorbereiten/zielen/sich zurückziehen auf, resultieren aus, kommen von; erkennen an, sich (er)freuen an, sich stören an, scheitern an, schwer tragen an, verzweifeln an).

Fakultativ ist ein Korrelat bei den dreiwertigen Verben der Handlungssteuerung, die bevorzugt mit Infinitivphrasen auftreten (ermahnen/ermuntern/überreden/ veranlassen/verführen zu). Dabei spielt die Tatsache eine Rolle, dass die Präpositionalobjektsätze bei diesen Verben Drittaktanten sind und ihre Identifizierung aus Verarbeitungsperspektive nach der Abarbeitung von Subjekt und Akkusativobjekt problemlos ist. Ferner haben diese Konstruktionen semantisch und in der Realisierung als Infinitiv eine Nähe zu kohärenten Konstruktionen mit lassen. Inwieweit der Umfang der Infinitivphrase (unerweitert vs. erweitert) mit der Akzeptabilität und Auftretenswahrscheinlichkeit korreliert, wäre noch zu verifizieren. 


\subsection{Strukturelle Bedingungen des Auftretens von Korrelaten}

Die funktionalen Vorzüge der Korrelatsetzung kommen auch in bestimmten strukturellen Umgebungen zum Tragen.

- Die Setzung des Korrelats erleichtert die Verarbeitung von Komplementsätzen in Stellungen, in denen sie ohne ein Korrelat schwer zu verarbeiten wären.

- Die Setzung des Korrelats erleichtert die Identifikation von Fokus und Fokusprojektion, also derjenigen Teile der Äußerung, die der Sprecher als zentrale Aussage verstanden wissen will. Das hat vor allem mit der Akzentrealisierung zu tun.

In beiden Fällen tritt die starktonige Form des Korrelats auf, die im Übrigen auch die einzige Form von Adverbialsatzkorrelaten ist (DEShalb, weil, DANN, wenn). Sie macht Präpositionalobjektsätze überhaupt erst vorfeld- und mittelfeldfähig. Komplementsätze im Vorfeld werden defaultmäßig als Subjekt- oder allenfalls als Akkusativobjekt interpretiert, sodass ein Präpositionalobjekt in dieser Position einer besonderen Kennzeichnung bedarf, nämlich einer Linksversetzungskonstruktion wie in (27a) (s. Altmann 1981). Im Mittelfeld, das generell nebensatzfeindlich ist, weil dann die Matrixsatzproposition erst nach Abarbeitung der Nebensatzproposition abgeschlossen werden kann, sind allenfalls solche Nebensätze erlaubt, die durch eindeutige morphologische Markierung in ihrer Relation zum Matrixsatz transparent sind: Bei Adverbialsätzen leistet dies der Subjunktor (weil, wenn, obwohl, statt dass etc.), bei Präpositionalobjektsätzen das Korrelat, das hier als Kopf einer Attributkonstruktion fungiert. Entsprechende Konstruktionen mit Subjekt- und Akkusativobjektsätzen sind deutlich weniger akzeptabel.

(27) a. Dass es ein sehenswertes Spiel wurde, dafür/^ sorgte auch das souveräne Schiedsrichtergespann. (Mannheimer Morgen, 19. 02. 2003)

b. Dass es ein sehenswertes Spiel wurde, ermöglichte auch das souveräne Schiedsrichtergespann.

(28) a. Die Türkei hat dafür, dass sie ihr Veto gegen den neuen Nato-Generalsekretär fallen liess, offenbar lukrative Zugeständnisse erhalten. (Die Südostschweiz, 06. 04. 2009)

b. ??Die Türkei hat das, dass sie ihr Veto gegen den neuen Nato-Generalsekretär fallen ließ, bezahlt bekommen.

Da die Bezugselemente zu Präpositionalobjektsätzen immer einen Akzent tragen, treten sie vor allem in Umgebungen auf, in denen der Komplementsatz durch Elemente wie Fokuspartikeln, Negation oder adverbiale skalare Ausdrücke fokussiert werden soll. 
(29) a. Wähler haben es darauf abgesehen, Politikern das Leben schwer zu machen.

b. Wähler haben es vor allem DArauf abgesehen, Politikern das Leben schwer zu machen.

\section{Topologische Eigenschaften von Präpositionalobjektsätzen}

Präpositionalobjektsätze sind auch in ihrem Stellungsverhalten im System der Komplementsätze auffällig. Die markantesten Unterschiede gegenüber Subjektund Akkusativobjektsätzen sind die in Abschnitt 3 erwähnte erhöhte Mittelfeldfähigkeit, die - außer bei freien Relativsätzen - an das Auftreten eines akzentuierten Korrelats im selben Stellungsfeld gebunden ist, und das Vorfeldverbot, das nur durch Konstruktionsmuster mit einem Korrelat kompensiert werden kann. Die Akzeptabilität von Komplementsätzen im Mittelfeld steigt mit dem Grad ihrer „Nominalität“ (Oppenrieder 2006: 909) und sinkt mit dem Grad der Assertiertheit: Mit abnehmender Wahrscheinlichkeit können hier auftreten: Freie Relativsätze < Infinitivphrasen < Verbletztsätze (dass-, $o b-, w$-) < Verbzweitsätze. Bei Freien Relativsätzen übernimmt das $w$-Relativum, dessen Form und Funktion zwar durch das Relativsatzprädikat selbst bestimmt wird, das aber mit der Funktion des Relativsatzes im Matrixsatz übereinstimmen muss, die Kennzeichnung der Funktion, sodass ein Korrelat überflüssig wird.

(30) Man sollte, wozu man selber nicht bereit ist, niemand anderen zwingen wollen.

In den meisten Fällen treten Präpositionalobjektsätze extraponiert auf, wobei im Mittelfeld ein Korrelat erscheinen kann bzw. - in Abhängigkeit von lexikalischen und strukturellen Faktoren - auftreten muss (s. Abschnitt 3.)

(31) a. Fromme verweist darauf, dass die niedersächsische Landesregierung sich im Bundesrat für die Kommunen eingesetzt habe. (Braunschweiger Zeitung, 18. 08. 2007)

b. Wenn der soziale Hintergrund nicht stimmt, kann man drauf warten, daß es auch in der Arbeit kracht. (Nürnberger Nachrichten, 12. 02. 1994, S. 3)

In seltenen Fällen sind Korrelate und Komplementsatz maximal distant, wenn nämlich das Korrelat im Vorfeld und der Komplementsatz am rechten Rand steht. Das Korrelat kann hier nicht schwachtonig oder reduziert sein (als Korrelat eines 
AkkO-Satzes kann hier ebenfalls nur das Pronomen das auftreten), der Präpositionalobjektsatz ist intonatorisch und mitunter auch interpunktorisch abgesetzt,

(32) Lästern stärkt das Wir-Gefühl: Darin/`Drin sind wir uns einig, dass die oder der andere komisch oder blöd ist. (http://www.ekkw.de/lekinet/predigten/ jahr56/5642.doc - 18. 02. 10)

Der Präpositionalobjektsatz in (32) ist in seiner Struktur ambig. Er kann als extraponierter Attributsatz zu einem pronominalen Kopf DArin gedeutet werden, aber auch als Nachtrag (darin sind wir uns einig, und zwar dass ...) gedeutet werden, wobei der dass-Satz dann eine Paraphrase für den vorhergehenden Satz Lästern stärkt das Wir-Gefühl wäre.

Korrelat und Präpositionalobjektsatz können auch gemeinsam rechts vom Matrixsatz erscheinen, was nach verbalen Valenzträgern deutlich seltener als nach Kopula und Adjektiv ist (erstaunt darüber, dass ..., begierig darauf, dass ... etc.). Das Stellungsmuster existiert für die akzentuierte wie für die unakzentuierte Variante des Korrelats. Im ersten Fall ist das Korrelat Fokusexponent für den voll fokalen Präpositionalobjektsatz und kann selbst durch eine Fokuspartikel oder kontrastierende Negation fokussiert sein wie in (33a), im zweiten Fall, der im Sinne der Verarbeitung als sukzessive Ausklammerung zu verstehen ist, wird umgekehrt das Matrixsatzprädikat fokussiert. In Bsp. (33b) und (33c) erfolgt dies vor dem Hintergrund eines Präpositionalobjektsatzes, dessen Inhalt im Vortext bereits thematisiert ist.

(33) a. Wer - wie vermutlich die Bundesregierung - dann prompt über die fortgesetzte Aushöhlung des Binnenmarkts lamentieren sollte, könnte nachdenken: [und zwar] zum Beispiel DAarüber, ob es nicht endlich an der Zeit wäre, sich für eine Abschaffung des Vetorechts in Sachen Steuern stark zu machen. (Frankfurter Rundschau, 13. 09. 1999, S. 11)

b. „Ich arbeite nicht gegen Mainz 05“, sagt der fußballverrückte CDU-Politiker gestern. Er habe sich „fürchterlich geÄRgert“ darüber, daß der Vereinsvorsitzende Harald Strutz dem für das Stadion und die Sportflächen zuständigen Grün- und Umweltdezernenten genau das unterstellt hatte.

c. Sehr gut! Danke! Ich hab schon geWARtet drauf, daß Du Bilder darüber postest.

(http://www.ureader.de/msg/114446456.aspx) [Akzentmarkierung EB]

Die Ausklammerung von Platzhalter und Komplementsatz ist ein Spezifikum von Präpositionalobjektsätzen, entsprechende Strukturen mit es sind ungrammatisch.

*Ich habe schon erWARtet es, dass du die Bilder rüber postest. 
Der Unterschied reflektiert unterschiedliche Valenzbindungsgrade, hinter denen letztlich ein Transitivitätsunterschied steht (vgl. Hopper/Thompson 1980, Ágel 2000: 126). Das Denotat eines Akkusativobjekts ist tendenziell stärker durch die Prädikatshandlung affiziert als das eines Präpositionalobjekts; das zeigen gerade Valenzträger mit alternierenden Valenzmustern (vgl. einen Brief schreiben vs. an einem Brief schreiben, etw. glauben vs. an etw. glauben). Je enger aber die Valenzbindung zwischen einer Konstituente und dem Prädikat, desto weniger lässt sie sich ausklammern. Kasuskomplemente erlauben Ausklammerung in aller Regel nicht, bei Präpositionalobjekten ist sie eher möglich.

Eine Abfolge Komplementsatz vor Matrixsatz ist deutlich seltener als die umgekehrte Abfolge. Sie kann dann günstiger sein, wenn der Inhalt des Komplementsatzes unmittelbar an den Vortext anknüpft und der Matrixsatz inhaltlich weitergeführt wird oder z.B. durch einen Adverbialsatz erweitert ist. Ein Präpositionalobjektsatz am linken Rand des Matrixsatzes kann in mehreren Konstruktionsmustern auftreten, ein Korrelat ist dabei immer obligatorisch.

(i) als Attributkonstruktion, bei der ein (akzentuiertes) Bezugselement zusammen mit dem nachfolgenden Präpositionalobjektsatz das Vorfeld besetzt oder aber gemeinsam linksversetzt ist. Diese Doppelmarkierung der Relation ist aus der Hörerperspektive vor allem bei sehr umfangreichen Komplementsätzen sinnvoll. (siehe 35a)

(ii) linksversetzt mit einem im Vorfeld stehenden Pronominaladverb. Bei diesem Stellungsmuster ist die reduzierte Form wohl nicht ganz auszuschließen, fraglich ist dann aber, ob es sich noch um eine Herausstellungsstruktur Linksversetzung handelt oder vielmehr Freies Thema vorliegt. [siehe 35c]

(iii) als Freies Thema im linken Außenfeld mit einem nur locker daran anschließenden Pronominaladverb im Mittelfeld des Matrixsatzes, wobei das Pronominaladverb akzentuiert oder unakzentuiert sein [siehe 35d]

(35) a. Dann gelte es, längere Fahrten in Etappen aufzuteilen.

Darauf/*drauf, dass Übersicht und Reaktionsvermögen nachlassen, weisen folgende Phänomene hin: Der Beifahrer sieht plötzlich mehr als der Fahrer, erkennt Gefahren früher und Situationen, in denen der Fahrer nicht hundertprozentig Herr der Lage ist, nehmen zu. (Mannheimer Morgen, 25. 08. 2007)

b. DArauf/^drauf, dass es den Wintercheck so lange gebe und er noch immer für die Wolfenbütteler kostenlos sei, darauf seien die Organisatoren stolz, so Gutsche. (Braunschweiger Zeitung, 14. 10. 2009)

c. Was fehlt, sind die Kinder. Dass sie ihre beiden Söhne wieder zu sich nehmen kann, darauf wartet sie noch immer. (Zürcher Tagesanzeiger, 10. 09. 1996, S. 10) 
d. Dass sie ihre beiden Söhne wieder zu sich nehmen kann - sie wartet noch IMmer d(a)rauf./?sie wartet DArauf noch immer.

\section{Zusammenfassung}

Präpositionalobjektsätze bilden einen weitgehend grammatikalisierten Bereich, der in seinen semantischen Rollen ein breites Spektrum zwischen zentralen und peripheren Komplementen abdeckt. In den zentralen Bereichen ähneln sie Akkusativobjektsätzen, in den peripheren adverbialen Nebensätzen. Die Eigenschaften von Präpositionalobjektsätzen ähneln damit gerade in ihrer Hybridität denen von PPen in PO-Funktion.

\section{Literatur}

Ágel, V. (2000): Valenztheorie. Tübingen: Narr.

Altmann, H. (1981): Formen der „Herausstellung“ im Deutschen. Rechtsversetzung, Linksversetzung, freies Thema und verwandte Konstruktionen. Tübingen: Niemeyer.

Bausewein, K. (1990): Akkusativobjekt, Akkusativobjektsätze und Objektsprädikate im Deutschen. Untersuchungen zu ihrer Syntax und Semantik. Tübingen: Niemeyer.

Beneš, E. (1979): Zur Konkurrenz von Infinitivfügungen und daß-Sätzen. In: Wirkendes Wort 29, 274-384.

Berman, J. (2003): Zum Einfluss der strukturellen Position auf die syntaktische Funktion der Komplementsätze. In: Deutsche Sprache 31, 263-286.

Boszák, G. (2009): Realisierung der valenzbestimmten Korrelate im Deutschen. Frankfurt/Main: Lang.

Breindl, E. (1989): Präpositionalobjekte und Präpositionalobjektsätze im Deutschen. Tübingen: Niemeyer.

Breindl, E. (2006): Präpositionalphrasen. In: Ágel, V./Eichinger, L.M./Eroms, H.W./Hellwig, P./ Heringer, H.J./Lobin, H. (Hgg.), Dependenz und Valenz. Ein internationales Handbuch der zeitgenössischen Forschung. 2. Halbband. Berlin: de Gruyter, 936-951.

Dal, I. (1966): Kurze deutsche Syntax auf historischer Grundlage. 5., verbess. Aufl. Tübingen: Niemeyer.

Engel, Ulrich (1977): Syntax der deutschen Gegenwartssprache. Berlin: Erich Schmidt Verlag.

Engel, Ulrich (1994): Syntax der deutschen Gegenwartssprache. 3., völlig neu bearb. Aufl. Berlin: Erich Schmidt.

Eroms, H.-W. (1981): Valenz, Kasus, Präpositionen. Untersuchungen zur Syntax und Semantik präpositionaler Konstruktionen in der deutschen Gegenwartssprache. Heidelberg: Winter. Eroms, H.-W. (2000): Syntax der deutschen Sprache. Berlin: de Gruyter.

Fabricius-Hansen, C. (1981): Was ist nun wieder ein Korrelat? Gedanken zur Rehabilitierung eines naiven Nebensatzbegriffs. In: Kopenhagener Beiträge zur germanistischen Linguistik 18, 1-45. 
Holmlander, I. (1979): Zur Distribution und Leistung des Pronominaladverbs als Bezugselement eines das Verb ergänzenden Nebensatzes/Infinitivs. Uppsala: Acta Universitatis Upsaliensis.

Hopper, P.J./Thompson, S.A. (1980): Transitivity in Grammar and Discourse. In: Language 56, 251-299.

Hundt, M. (2001): Grammatikalisierungsphänomene bei Präpositionalobjekten in der deutschen Sprache. In: Zeitschrift für Germanistische Linguistik 29, 167-191.

Jacobs, J. (1994): Kontra Valenz. Trier: Wissenschaftlicher Verlag.

Oppenrieder, W. (1991): Von Subjekten, Sätzen und Subjektsätzen. Untersuchungen zur Syntax des Deutschen. Tübingen: Niemeyer.

Oppenrieder, W. (2006): Subjekt- und Objektsätze. In: Ágel, V./Eichinger, L.M./Eroms, H.W./ Hellwig, P./Heringer, H.J./Lobin, H. (Hgg.), Dependenz und Valenz. Ein internationales Handbuch der zeitgenössischen Forschung. 2. Halbband. Berlin: de Gruyter, 900-912.

Paranhos Zitterbart, J. (2002): Zur korrelativen Subordination im Deutschen. Tübingen: Niemeyer.

Pasch, R. (1977): Zum Status der Valenz. In: Linguistische Studien Reihe A 42, 1-50.

Pittner, K. (1995): The Case of German Relatives. In: The Linguistic Review 12, 197-232.

Pittner, K. (2003): Kasuskonflikte bei freien Relativsätzen - Eine Korpusstudie. In: Deutsche Sprache 31, 193-208.

Reis, M. (1997): Zum syntaktischen Status unselbstständiger Verbzweit-Sätze. In: Dürscheid, C./ Ramers, K.H./Schwarz, M. (Hgg.), Sprache im Fokus. Festschrift für Heinz Vater zum 65. Geburtstag. Tübingen: Niemeyer, 130-144.

Roustila, J. (2005): Zur Grammatikalisierung von Präpositionalobjekten. In: Leuschner, T./ Mortelmans, T./de Groodt, S. (Hgg.), Grammatikalisierung im Deutschen. Berlin: de Gruyter, 135-167.

Schumacher, H./Kubczak, J./Schmidt, R./de Ruiter, V. (2004): VALBU - Valenzwörterbuch deutscher Verben. Tübingen: Narr [E-VALBU: http://hypermedia2.ids-mannheim.de/evalbu/ index.html.]

Steinitz, R. (1969): Adverbial-Syntax. Berlin: Akademie Verlag.

Sonnenberg, B. (1992): Korrelate im Deutschen. Beschreibung, Geschichte und Grammatiktheorie. Tübingen: Niemeyer.

Storrer, A. (1992): Verbvalenz. Theoretische und methodische Grundlagen ihrer Beschreibung in Grammatikographie und Lexikographie. Tübingen: Niemeyer.

Tesnière, L. (1959): Éléments de syntaxe structurale. Paris: Klincksieck.

Wolf, N.-R. (2003): Ebenen der Valenzbeschreibung: Die syntaktische Ebene. In: Ágel, V./Eichinger, L.M./Eroms, H.W./Hellwig, P./Heringer, H.J./Lobin, H. (Hgg.), Dependenz und Valenz. Ein internationales Handbuch der zeitgenössischen Forschung. 1. Halbband. Berlin: de Gruyter, 404-410.

Zifonun, G./Hoffmann, L./Strecker, B. et al. (1997): Grammatik der deutschen Sprache. 3 Bde. Berlin: de Gruyter.

Zint-Dyhr, I. (1981): Ergänzungssätze im heutigen Deutsch. Untersuchungen zum komplexen Satz. Tübingen: Narr. 